\title{
Adaptive PF Speed Control of SRM Drives
}

\author{
Laszlo Szamel \\ Budapest University of Technology and Economics /Department of Electric Power Engineering, \\ Budapest, Hungary, e-mail: szamel@eik.bme.hu
}

\begin{abstract}
This paper proposes two model reference adaptive PF speed control methods for SRM drives. Following from the structure of model reference parameter adaptive PF control it makes it easier to reach overshootless as well as fast speed changing compensation caused by jump in load. The approaching block diagram of the model reference signal adaptive control can be seen as an extended version of the PF controller, so one of the adaptation factors (which is the free parameter of the adaptive control) is given. Both model reference adaptive controls drawn up can be easily implemented because the adaptation algorithms do not need acceleration measuring.
\end{abstract}

Keywords-Adaptive control, Control of Drive, Servodrive, Switched reluctance drive.

\section{INTRODUCTION}

In motion control systems there is robustness against parameter changes and disturbance rejection of main interest. The model reference adaptive control has the following features:

- It enables the compliance of the system with varying operational conditions and ensures the behavior of the controlled system according to the prescribed reference model.

- It means such a special type of adaptive systems which results in nonlinear control systems. This is the reason why the analytical analysis is completed by the Ljapunov stability criterion or by the hyper-stability principle.

- Its design and application are closely related to the using of computer methods.

- Simple implementation of the control algorithm.

In this paper applications of two model reference adaptive control methods to switched reluctance motors are presented.

\section{DRIVE SYSTEM}

Block scheme of the examined drive system is shown in Fig. 1.

The supply unit consists of three main blocks, namely the RECTIFIER, the FILTER and the INVERTER. The inverter is a pulsed width modulated (PWM) one, marked by $\mathrm{QP}$ in the figure and it contains a one-one switching transistor per phase and a brake chopper, not shown in the figure. The common point of phase windings is supplied by the PWM inverter. It is of autonomous operation and has an inner current control loop. The other ends of phase windings are connected to the phase switching transistors.

It follows from the operational principle of SRM that its phase windings are to be excited at a well determined angle of the rotor position in an appropriate order. That is why a Rotor Position Sensor is to be mounted on the shaft of the motor. In our case the position sensor is a resolver. It can be calculated from the pole numbers that the phase switchings have to follow each other by 15 degrees. The resolver is supplied by an oscillator circuit, their signals are evaluated by a Position Decoder.

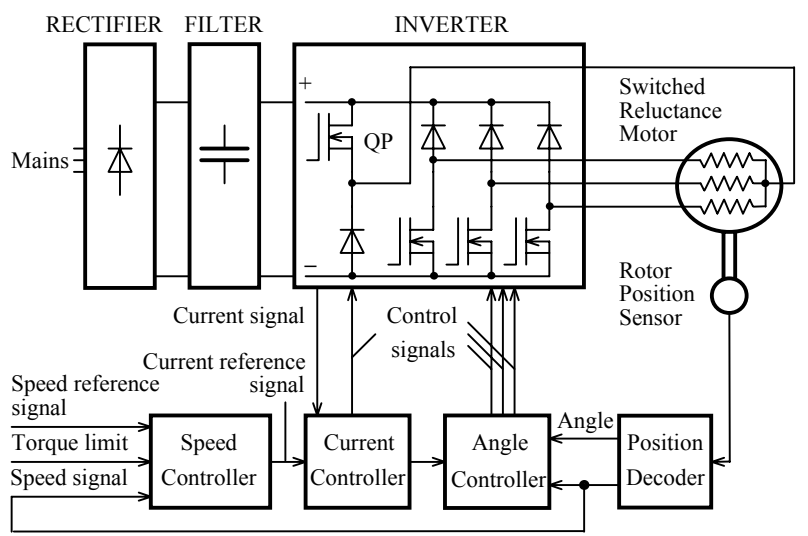

Fig. 1. Block scheme of drive system

The Position Decoder has two outputs: Angle and Speed signals. Angle Controller provides the Control signals for the phase switching transistors based on the two signals.

\section{CURRENT CONTROL}

The Current Controller is totally based on its hardware solution. Based on the current reference signal it controls the PWM inverter of fixed frequency by installing an analog controller. The current feedback also comes from the PWM inverter.

For the control of the sum of phase currents (Fig. 1) a simpler four-transistor inverter is suitable and a sixtransistor one is not necessary as in the case of control of phase currents independently from each other. But the detriment of the previous solution is that torque pulsation can be decreased in a smaller degree by changing turn-on and turn-off angles.

Namely in case of constant current reference signal the current increase is limited by the switched-off, but the conducting phase current as regulator controls the sum of two phase currents. The increase of phase current at the beginning of the conducting state can be forced by the modification of the current reference signal:

$$
i_{r}=u \sum_{j=1}^{3} C_{j}+\sum_{j=1}^{3}\left(1-C_{j}\right) \cdot i_{j} .
$$

where:

$i_{r} \quad$ is the current reference signal, 
$i_{j} \quad$ is the current signal of phase $j$,

$u \quad$ is the output of the speed controller,

$C_{j} \quad$ is the control signal of phase $j$ ( 0 or 1$)$.

Supplement of the first member in (1) makes the overlap of the phase conduction possible, while the effect of the second member is to increase the reference signal with the current of the switched-off but not current-free phase.

\section{SPEED CONTROL}

A model reference adaptive control is used for speed control. Such an adaptive control has been successfully elaborated by using a suitable chosen Ljapunov function to compensate the gain of the speed control loop [1], [2], [6], [7].

\section{A. Model Reference Parameter Adaptive Control}

The adaptive control of servo-drives with a cascade arrangement is most effective when it is applied in the inner loop containing the effect of variable parameters directly, i.e. inertia $\left(J_{m}\right)$ and/or torque factor $\left(k_{m}\right)$. The speed control implemented by PI controller is of a cascade arrangement in fact as it contains an inner, proportional feedback loop (PF controller, [4], Fig. 2). A one-storage proportional element can describe this inner loop neglecting the time constant of closed current control loop. By this our adaptation algorithm is simplest.

Relation between accelerating current determining dynamic torque and angular speed can be given by the following transfer function:

$$
Y_{\omega,\left(i-i_{t}\right)}(s)=\frac{A_{i}}{s}
$$

where: $A_{i}=\frac{k_{m}}{J_{m}}$.

Arrangement of control circuit can be seen in the following figure:

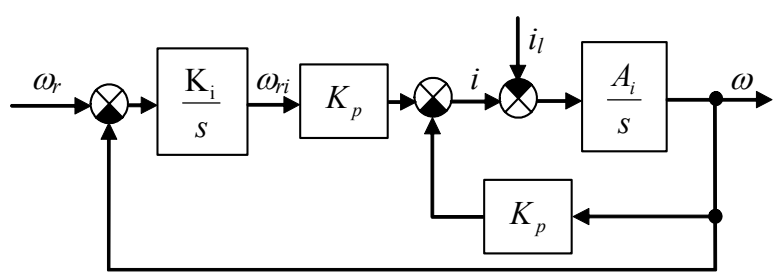

Fig. 2. Block scheme of parameter adaptive PF speed control

where

$\omega$ is the speed,

$\omega_{r} \quad$ is the speed reference signal,

$i \quad$ is the current of the motor,

$i_{l} \quad$ is the current equivalent to the load-torque.
Section determined by the transfer function $Y_{\omega,\left(i-i_{l}\right)}(s)$ is fed back by a proportional member of gain $K_{P}$. The task is to change gain $K_{P}$ in such a way that the product $A_{i} K_{P}$ should remain constant despite the changing of $A_{i}$ parameter.

Transfer factor of the inner closed loop is given by the reciprocal $\left(1 / K_{P}\right)$ of feedback member that is not constant because of the change in torque factor. Consequently loop gain of outer speed control loop would change as well. In order to get a one-storage element with unity transfer factor we have to insert a member with gain $K_{P}$ between the integrator of the PF controller and the reference signal of the inner loop. First-order reference model with time constant $T_{m}$ gets sum of the input signals of above member $\left(\omega_{r i}\right)$ and signal $\omega_{l m}$ compensating the load effect for the model. So dynamics of reference model can be described by the following differential equation:

$$
\dot{\omega}_{m} T_{m}+\omega_{m}=\omega_{r i}+\omega_{l m}
$$

Dividing (3) with $T_{m}$ and applying designation $q_{m}=1 / T_{m}$, we get the following equation.

$$
\dot{\omega}_{m}+q_{m} \omega_{m}=q_{m}\left(\omega_{r i}+\omega_{l m}\right) .
$$

The differential equation of the first-order controlled plant is as follows:

$$
\dot{\omega}+\left(K_{p} A_{i}\right) \omega=\left(K_{p} A_{i}\right) \omega_{r i}-A_{i} i_{l} .
$$

Factor $K_{p}$ can be described as the sum of $K_{p 0}$ determined by mean $A_{i}$ and $\Delta K_{p}$ accomplished by the adaptation algorithm. So:

$$
K_{p} A_{i}=\left(K_{p 0}+\Delta K_{p}\right) A_{i}=q+\Delta q,
$$

where $K_{P 0}$, and $q$ are constant.

In this case we assume that the change of $A_{i}$ is slow from the viewpoint of adaptation and therefore the effect of this change can be neglected.

Substituting (6) into (5) we get:

$$
\dot{\omega}+(q+\Delta q) \omega=(q+\Delta q) \omega_{r i}-A_{i} i_{l} .
$$

By using (4) and (7) and substituting expression of model error $\varepsilon=\omega_{m}-\omega$ the dynamic equation will be:

$$
\dot{\varepsilon}=-q_{m} \varepsilon+x \omega-x \omega_{r i}+q_{m} \omega_{l m}+A_{i} i_{l},
$$

where $x=(q+\Delta q)-q_{m}$.

Dynamic of model error should by asymptotically stable to follow the system with model proposed. For determination of $\Delta q$ the following Ljapunov function should be composed:

$$
V=\frac{1}{2}\left(\varepsilon^{2}+\beta x^{2}\right)
$$

where $\beta$ is a positive number. 
When choosing the Ljapunov function both purposes, i.e. the termination of the model error and loop gain deviation have been taken account.

The time derivative of the Ljapunov function is:

$$
\dot{V}=\varepsilon \dot{\varepsilon}+\beta x \dot{x}
$$

Substituting (8) into (10) the following equation is valid:

$$
\begin{aligned}
& \dot{V}=-q_{m} \varepsilon^{2}+\varepsilon x \omega-\varepsilon x \omega_{r i}+ \\
& \varepsilon\left(q_{m} \omega_{l m}+A_{i} i_{l}\right)+\beta x \dot{x} .
\end{aligned}
$$

If

$$
\varepsilon \times \omega-\varepsilon \times \omega_{r i}+\beta x \dot{x}=0,
$$

that is

$$
\dot{x}=\varepsilon\left(\omega_{r i}-\omega\right) / \beta
$$

and

$$
\varepsilon\left(q_{m} \omega_{l m}+A_{i} i_{l}\right)<0
$$

then

$$
\dot{V}<-q_{m} \varepsilon^{2} .
$$

The above equation is a negative definite function that shows the asymptotic stability of the error dynamic (8). By using (6), (8) and (13) the following adaptation algorithm is true:

$$
\Delta \dot{K}_{p}=\gamma \varepsilon\left(\omega_{r i}-\omega\right) .
$$

where $\gamma$ may be an arbitrary positive number. The inequality (14) shows how we have to change the signal $\omega_{l m}$ representing the load of model.

If

$$
\varepsilon>0, \text { then } \omega_{l m}<-\frac{\left|i_{l}\right|_{\max } A_{i}}{q_{m}},
$$

respectively if

$$
\varepsilon<0, \text { then } \omega_{l m}>\frac{\left|i_{l}\right|_{\max } A_{i}}{q_{m}} .
$$

\section{B. Model Reference Signal Adaptive Control}

The controlled loop has been approximated by an integral element. Time constant of the closed current control loop has been neglected. The control consists of a P-element with the gain $K_{p}$. Input of P-element contains not only the control error signal but an adaptation signal as well $(g)$. Applying the signal adaptation control, a P type controller with $K_{p}$ gain can ensure zero speed error as the adaptation signal can produce a current reference signal to compensate the loading current at zero speed error.

The feature of closed speed control loop has been taken into consideration by a parallel control model to be expressed by first order proportional element. The Ljapunov function has been chosen in such a way that model error should be decreased asymptotically and gain of speed control loop and load should be compensated. In this case we have to assume that change in loop gain and in load is smaller than the speed of the adaptation.

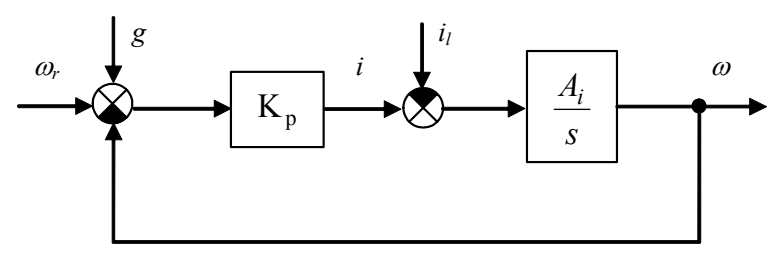

Fig. 3. Initial block scheme of signal adaptive speed control

Regarding the block diagram of control loop following differential equation is valid for the closed loop:

$$
\dot{\omega}+A_{i} K_{p} \omega=A_{i} K_{p}\left(\omega_{r}+g\right)-A_{i} i_{l} .
$$

The feature of the closed speed control loop has been taken into consideration by a parallel control model to be expressed by a first order proportional element. The differential equation of the first order system is:

$$
\dot{\omega}_{m}+q_{m} \omega_{m}=q_{m} \omega_{r} .
$$

where index $\mathrm{m}$ refers to the model and $q_{m}$ is the reciprocal of model time constant.

Using (18) and (19) and introducing the expression $\varepsilon=\omega_{m}-\omega$ for model error, the dynamic equation for the model error is as follows:

$$
\dot{\varepsilon}+q_{m} \varepsilon=\left(q_{m}-A_{i} K_{p}\right)\left(\omega_{r}-\omega\right)+A_{i}\left(i_{l}-K_{p} g\right) .
$$

The adaptation signal $g(t)$ can be written in the following form:

$$
g(t)=g_{1}(\mathrm{t})\left(\omega_{r}-\omega\right)+g_{2}(t) .
$$

Substituting (21) for (20):

$$
\dot{\varepsilon}=-q_{m} \varepsilon+b_{1}\left(\omega_{r}-\omega\right)+b_{2},
$$

where

$$
\begin{gathered}
b_{1}=q_{m}-A_{i} K_{p}\left(1+g_{1}(t)\right), \\
b_{2}=A_{i}\left(i_{l}-K_{p} g_{2}(t)\right) .
\end{gathered}
$$

Let us compose the following Ljapunov function to produce the signal $g_{1}(t)$ and $g_{2}(t)$ :

$$
V=\frac{1}{2} \varepsilon^{2}+\frac{1}{2}\left(\beta_{1} b_{1}^{2}+\beta_{2} b_{2}^{2}\right),
$$

where $\beta_{1}$ and $\beta_{2}$ are positive constants.

Time-derivation of the Ljapunov function is:

$$
\dot{V}=\varepsilon \dot{\varepsilon}+\beta_{1} b_{1} \dot{b}_{1}+\beta_{2} b_{2} \dot{b}_{2} .
$$

Substituting (22) for (24): 


$$
\begin{aligned}
\dot{V}= & -q_{m} \varepsilon^{2}+\left(\omega_{r}-\omega\right) b_{1} \varepsilon+b_{2} \varepsilon \\
& +\beta_{1} b_{1} \dot{b}_{1}+\beta_{2} b_{2} \dot{b}_{2} .
\end{aligned}
$$

If

$$
\dot{b}_{1}=-\left(\omega_{r}-\omega\right) \varepsilon / \beta_{1}
$$

and

$$
\dot{b}_{2}=-\varepsilon / \beta_{2}
$$

then

$$
\dot{V}=-q_{m} \varepsilon^{2}
$$

and it ensures asymptotical stability of the model error. On the basis of (22), (26) and by assuming that variation of $A_{i}$ can be neglected compared to the speed of adaptation the following adaptation algorithm is valid:

$$
\begin{gathered}
\dot{g}_{1}(t)=\gamma_{1} \varepsilon\left(\omega_{r}-\omega\right), \\
\dot{g}_{2}(t)=\gamma_{2} \varepsilon .
\end{gathered}
$$

where $\gamma_{1}$ and $\gamma_{2}$ are positive constants, the free parameters of adaptation. Taking relations (21), (28) into consideration the following equation comes true:

$$
g(t)=\gamma_{1}\left(\omega_{r}-\omega\right) \int \varepsilon\left(\omega_{r}-\omega\right) d t+\gamma_{2} \int \varepsilon d t .
$$

A block diagram of the control circuit introducing adaptation signal $g(t)$ furthermore $g_{1}(t)=$ const. can be seen in Fig. 4. Taking the structure of control: it contains two parts. In the first part the reference signal is led through a first order system and a PI controller with variable gain and integration time. The second one is a differentiating filter which takes effect only on changing of reference signal. The gain and differentiation time are also changing. The adaptation gain factor $\gamma_{2}$ gives the reciprocal of integrating time constant of controller type PI, assuming $g_{1}(t)=0$. To fulfill the constant integrating time constant it is preferable to substitute $\gamma_{2}$ by $\gamma_{2}\left(1+g_{1}(t)\right)$. In such a way the neglect of time constant of current control loop can be compensated.

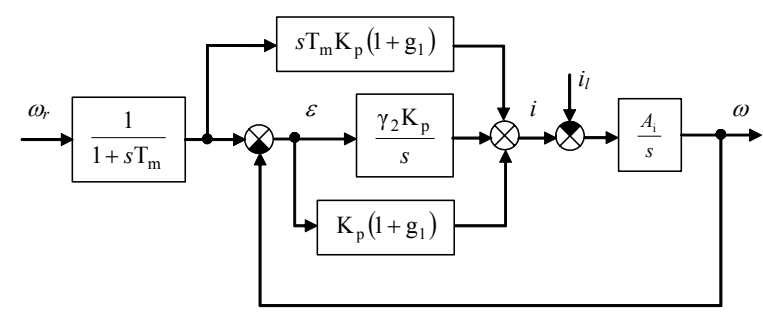

Fig. 4. Block scheme of the signal adaptive speed control with adaptation signal

The approaching block diagram of the adaptive control can be seen as an extended version of the PF (integral element with separate proportional feedback loop, [3], [6], the input of the proportional element is only the speed) controller so $\gamma_{2}$ one of the adaptation factors (which is the free parameter of the adaptive control) is given. Contraction of model-filtered reference signal and PI controller can be transformed into a PF controller when integration time of PI controller equals the time constant of the model (Fig. 5).

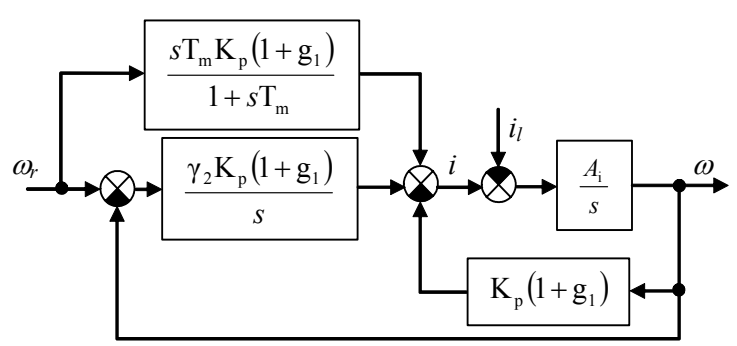

Fig. 5. Block scheme of signal adaptive PF speed control (extended version)

The basic structure of the signal adaptive speed controller is also PF type which on the one hand provides overshootless with its structure. Moreover, it ensures fast compensation of speed variation caused by a jump in motor load.

This control has been tested by a simulation program developed in our Department. First the adaptation has been examined without current limitation and load in order to take into consideration only the nonlinearity of the motor and the adaptation. In the interest of the adaptation stability speed of change of adaptation signal $g_{1}$ has to be limited. The signal $g_{1}$ results in significant oscillations without limitations as the change of the signal is possible in discrete times.

The current limitation causes further problems. This limitation hinders the tracking of the model so the effect of above signal $g_{1}$ will be too large or it can change in the reverse direction. To eliminate the above problem signal $g_{1}$ is not to be changed in the period of the current limitation.

Digital output signal determined by software is converted by a D/A converter to an analog reference signal. Speed feedback signal is determined by calculating the difference between the actual position value and previous one of the resolver to digital converter. The measuring period ensures an accuracy of $\pm 5 \mathrm{rpm}$.

\section{RIPPLE REDUCED CONTROL METHOD}

Ripple free operation of the SRM drives can only be realized with current waveform depending on rotor angle, speed and torque [5]. Ripple reduced method proposed changes only the turn-on and the turn-off angle in function of speed and current reference. Optimum turn-on and turnoff angles of SRM drive have been determined by computer simulation based on measured results of the analyzed drive. An optimum solution has been fulfilled by four cycles embedded into each other. Two outer cycles give the current and speed reference signals, while two inner ones provide the turn-on and turn-off angles. By this one-one optimum angle pair can be determined to all operating points.

It can be considered as an interesting result that the criterion of minimal torque pulsation does not provide an 
optimum solution in all cases. The torque pulsation will be minimum in the speed-current plane only in those cases when torque of the motor is relatively small. For this reason a good result can be achieved if the relative, i.e. compared to the torque of motor, torque pulsation is minimized.

The angle control of the drive determines actual turn-on and turn-off angles with a two-variable interpolation from the results stored in a look-up table and calculated by the above method.

\section{Simulation RESULTS}

According to the simulation investigations convergence of the model reference signal adaptive control at switched reluctance drives with significant torque ripples can be ensured under the following conditions:

- Input of the first-order reference model only determines its output when the filtered current reference signal is lower with a given $\Delta \mathrm{I}$ value (in the simulation it has been set to $1 \mathrm{~A}$ ) than its limit otherwise output is identical to the speed feedback signal. So the model works like a first-order proportional lag element only in operation without current limitations.

- Adaptation is executed when two conditions are true at the same time: filtered current reference signal is lower with a given $\Delta \mathrm{I}$ value (in the simulation it has been set to $1 \mathrm{~A}$ ) than current limit and absolute value of the speed error signal is higher than a given value (in the simulation it has been set to 20 revolution/ $\mathrm{min}$ ).

- The appropriate selection of the adaptation factors also has an important effect on the sufficient convergence.

Because of the restrictions described in point 2 . adaptation works practically only in a relatively narrow speed error track (adaptation range) which is equal to approximately $20-100 \mathrm{revolution} / \mathrm{min}$ absolute value of speed error. The drawback of this limitation is the relatively short time for the algorithm to operate. At the same time the convergence of the algorithm is extremely fast which significantly reduces the effect of this drawback. Two more important advantages emerge when adaptation works only with small speed errors. First of all the controller at changing drive parameters adapts to parameters around the value specified by the speed reference signal which also assists to speed the adaptation. The other significant positive effect is the disappearance of the problem coming from nonlinear systems that the response of the system can even differ in its character when the value, amplitude of the reference signal is changed.

In Fig. 6 and Fig. 7 two of many executed simulations are shown. Fig. 6 shows the run-up with speed controller of PF-type (an integral element with Proportional Feedback), while Fig. 7 with model reference parameter adaptive control (16), (17) and in both cases with turn-on and turn-off angles depending on speed and current reference and with current reference compensation (1). Fig. 8 shows the run-up with model reference signal adaptive speed controller and with jump in load.

\section{RESULTS}

The tests were completed by the described drive system. The test results have supported our theoretical investigations. The oscillograms in the following figures illustrate some typical starting curves and wave forms. The loading machine was a DC motor. Its inertia is about a triple of that of SRM.

Fig. 9 and Fig. 10 show the speed and current curves in the course of starting without current reference compensation.

The upper curve is the speed (1500 rpm), the lower one is the current flowing in the common point of stator windings $(10 \mathrm{~A} / \mathrm{div})$. It is related to the no-load operation mode.

The experiences show that the model reference parameter adaptive and signal adaptive control suggested in this paper works without overshooting.

\section{VIII.CONCLUSIONS}

The paper proposes a simple control method for SRM drives. The proposed ripple reduced control method changes only the turn-on and the turn-off angles depending on the speed and current reference. The modification of the current reference is suggested for a simpler four-transistor inverter.

To provide constant loop gain in speed control loop with changing parameters (moment of inertia and/or torque factor), parameter and signal adaptive model reference adaptive control was developed.

Following from the structure (PF-type) of model reference parameter adaptive control was developed to provide constant loop gain in speed control loop with changing gain (moment of inertia and/or torque factor) it makes it easier to reach overshootless as well as fast speed changing compensation caused by jump in load. The algorithm even keeps its stability at fast changing, jumplike load torque.

Model reference signal adaptive control is used to provide constant loop gain in speed control loop with changing parameters (moment of inertia and/or torque factor) exposed to a significant load. The approaching block diagram of the adaptive control can be seen as an extended version of the PF controller, so one of the adaptation factors (which is the free parameter of the adaptive control) is given.

With appropriate choice of the adaptation range convergence of the adaptation can even be ensured at SRM drives with significant torque ripples. Although normally because of the nonlinear characteristic of the adaptation, the response of the system also depends on the value of the jump in the reference signal, this does not cause any problems in the proposed adaptive control as adaptation only happens in a narrow track of the reference signal called the adaptation range.

The adaptive controls suggested in this paper work without overshooting. Though these methods require a longer calculation period it is less sensitive to the variations of parameters. Both model reference adaptive controls drawn up can be easily implemented, because the adaptation algorithms do not need acceleration measuring (thanks to the first-order model). Simulation and experimental results demonstrate that the proposed methods are promising tools to control SRM drives. 

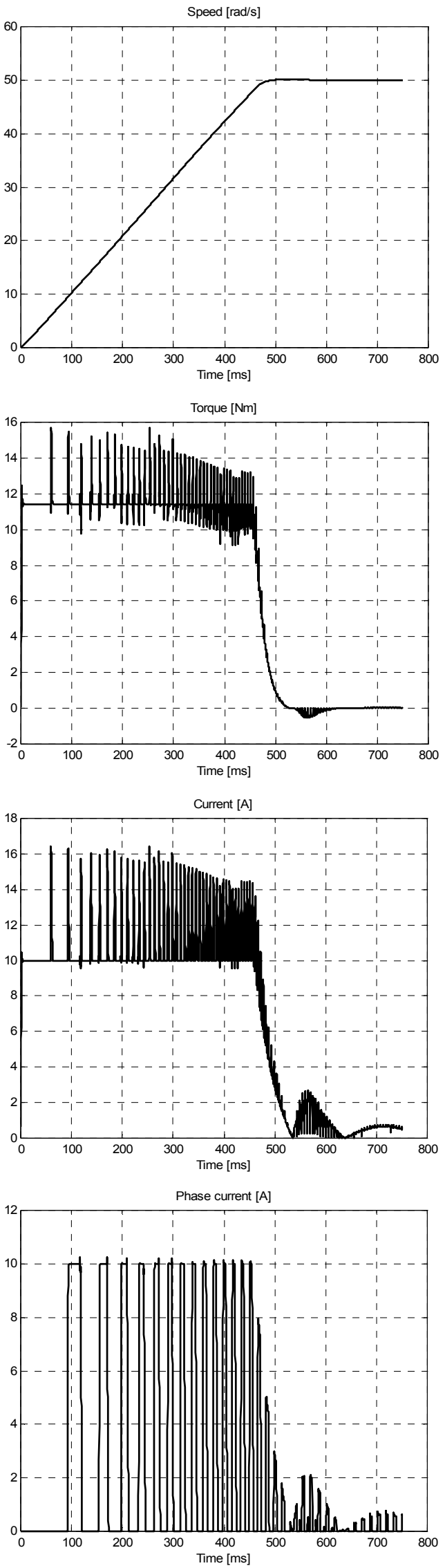

Fig. 6. Simulation results with PF-type speed control $\left(\Delta K_{p}=0\right)$
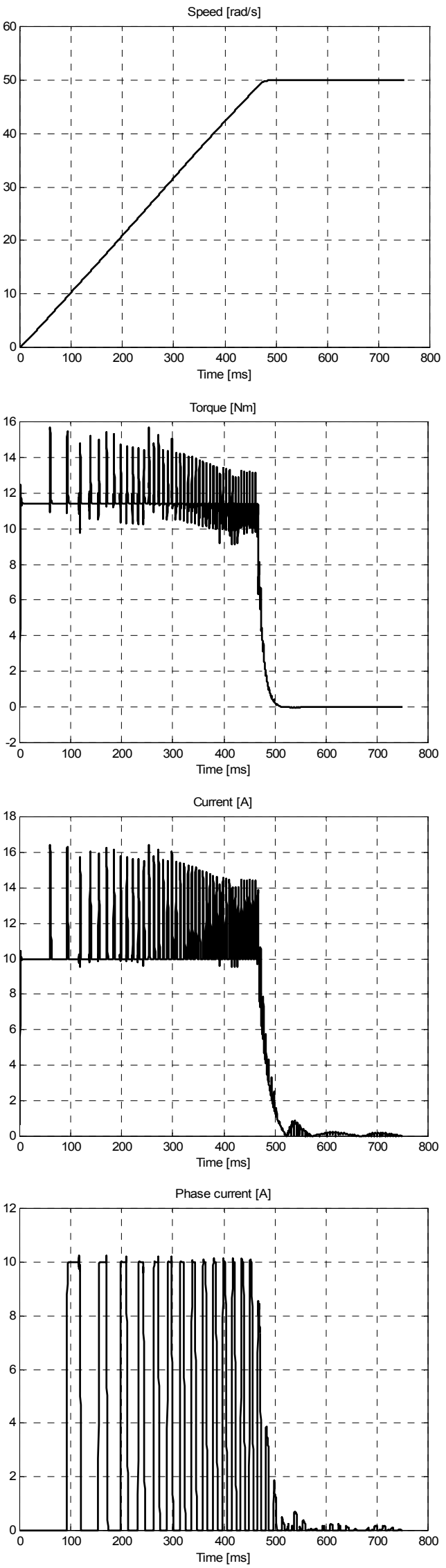

Fig. 7. Simulation results with model reference parameter adaptive speed control 

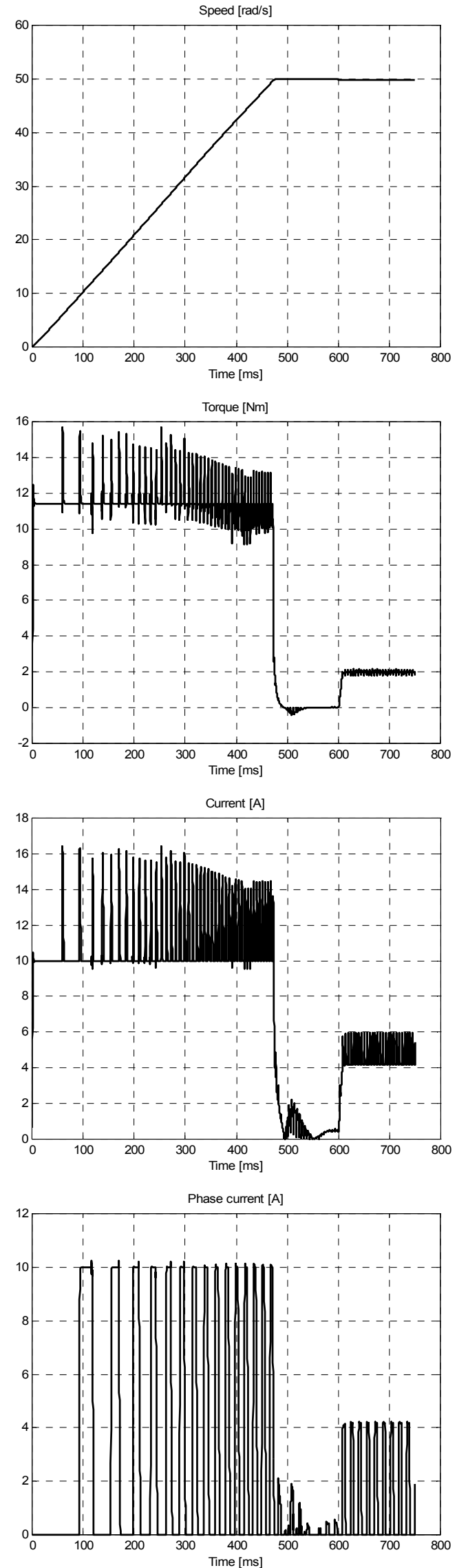

Fig. 8. Simulation results with model reference signal adaptive speed control

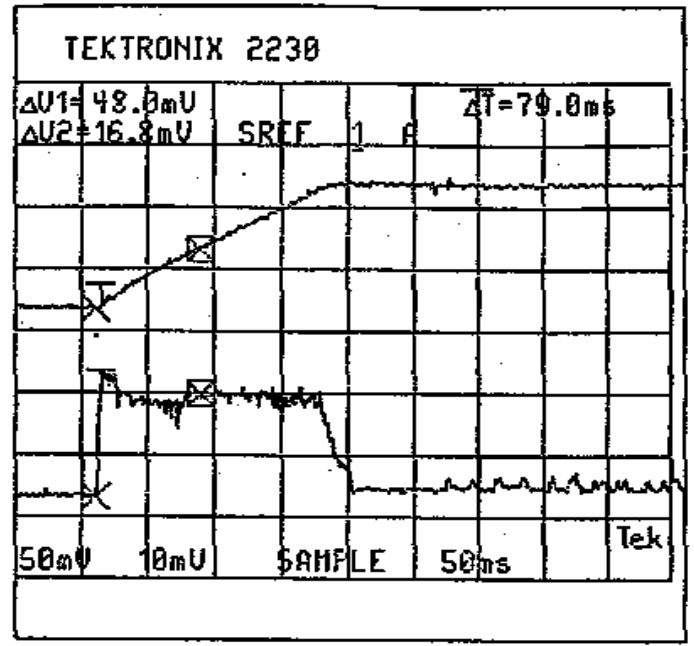

Fig. 9. Oscillogram of speed and current with model reference parameter adaptive speed control

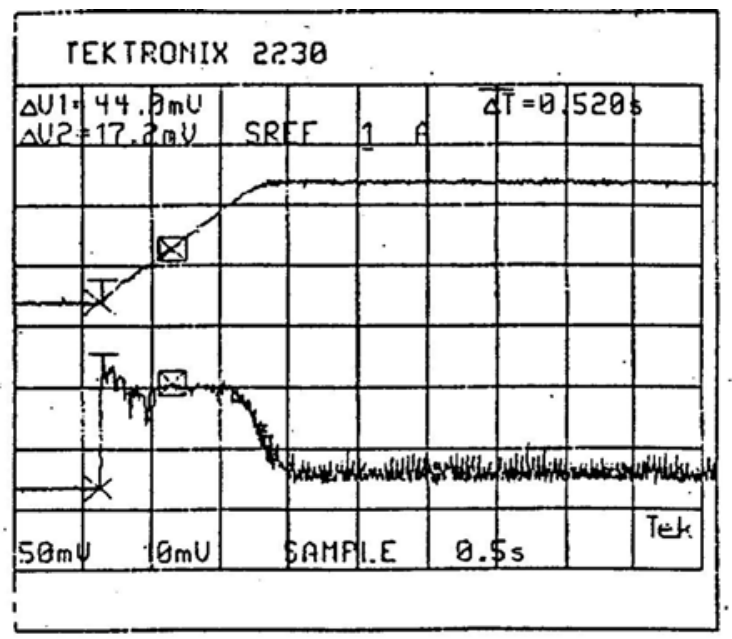

Fig. 10. Oscillogram of speed and current with model reference signal adaptive speed control

\section{REFERENCES}

[1] J. Borka, and L. Szamel, "Modern Strategy for Controlling Robot Drives," Conference Automation'92, Budapest, 1992, pp. 392401.

[2] B. K. Bose, and T. G. E. Miller, "Microcomputer Control of Switched Reluctance Motor," IEEE/IAS Annual Meeting, 1985, pp. 542-547.

[3] N.V. Diep, and L. Szamel, "Up-to-date Control Strategy in the Regulators of Robot Drives," PEMC'90, Budapest, 1990, pp.811815.

[4] G. A. Perdikaris, and K. W. VanPatten, "Computer Schemes for Modeling, Tuning and Control of DC Motor Drive Systems," PCI Proc., Mar. 1982, pp. 83-96.

[5] A. Stankovic, and G. Tadmor, "On Torque Ripple Reduction in Current-Fed Switched Reluctance Motors," IEEE Transactions on Industrial Electronics, Vol. 46, No. 1, February 1999. pp. 177-183

[6] L. Szamel, "Adaptive Control of SRM Drives," EPE-PEMC 2002 , Cavtat \& Dubrovnik (Croatia), 9-11 September 2002, CD-ROM: Paper No. T11-007.

[7] L. Szamel, "Investigation of Model Reference Parameter Adaptive SRM Drives," EPE-PEMC 2004, Riga (Latvia), 2-4 September 2004, Full paper A95117 on CD ROM. 